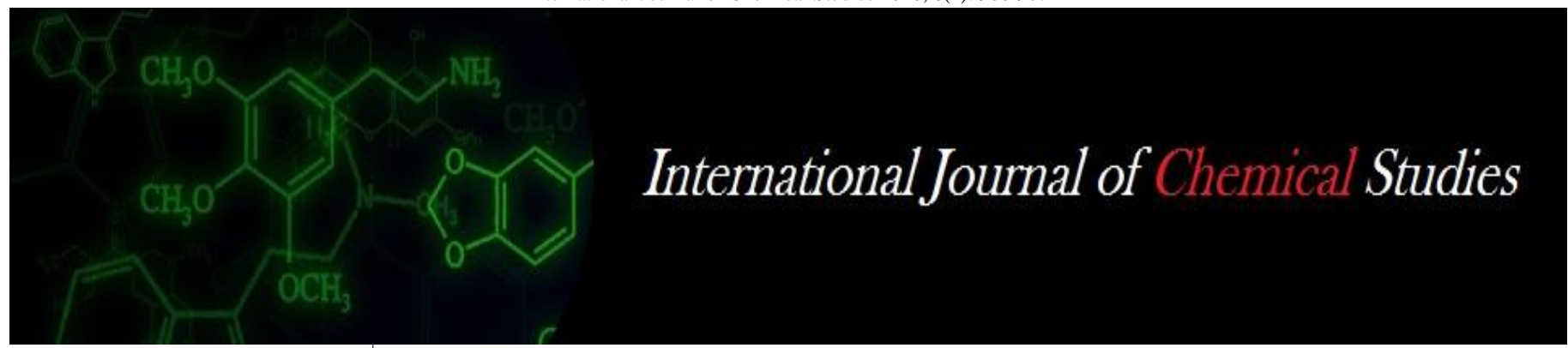

P-ISSN: 2349-8528

E-ISSN: 2321-4902

www.chemijournal.com

IJCS 2020; 8(4): 906-907

(C) 2020 IJCS

Received: 10-05-2020

Accepted: 12-06-2020

Irfan Ahmad Siddique

Research Scholar, Dept. of

Chemistry, L.N. Mithila

University, Darbhanga, Bihar,

India

\section{Studies of mixed ligand complexes of rubidium and caesium metal salts of some organic acids}

\section{Irfan Ahmad Siddique}

DOI: https://doi.org/10.22271/chemi.2020.v8.i4f.9719

\section{Abstract}

The present study was aimed at synthesised and characterised complexes of $\mathrm{Rb}$ and $\mathrm{Cs}$ metal salts of some organic acids, viz. o-nitrophenol, 2, 4-dinitrophenol, 1-nitroso-2-naphthol \& 8-hydroxy quinoline with o-aminothiophenol having a coordination sites $\mathrm{N}$ and $\mathrm{S}$ atoms.

Keywords: caesium metal, rubidium, ligand

\section{Introduction}

Complexes of transition metals[1-3] as well as non-transition metals[4-5] with systems containing both nitrogen and sulphur donors have been reported. In this paper, Complexes of the type ML.HL', have been synthesised and characterised, where $\mathrm{M}=\mathrm{Rb}$ or $\mathrm{Cs}, \mathrm{HL}$ ' = oaminothiophenol, L=deprotonated o-nitrophenol (ONP), 2, 4- dinitrophenol (DNP), 1nitroso-2-naphthol (1N2N) \& 8-hydroxy quinolin (8HQ). All the complexes have been found to be four coordinated in which ligand $\mathrm{O}$-aminothiophenol is coordinated through $\mathrm{N}$-atom of amino $\left(\mathrm{NH}_{2}\right)$ group \& $\mathrm{S}$-atom of the thiol $(-\mathrm{SH})$ group forming five membered stable chelation.

\section{Material and Methods}

1-Nitroso-2-naphthol (1N2N), o-nitrophenol (ONP), 2, 4-dinitrophenol (DNP) and 8hydroxyquinoline (8HQ) of Anal R grade were taken, $\mathrm{O}$-aminothiophenol of E.M. grade was distilled at $23^{\circ} \mathrm{C}$ and when cooled, its needle like crystals (m.p. $26^{\circ} \mathrm{C}$ ) were obtained Equimolar proportion of $\mathrm{RbOH}$ or $\mathrm{CSOH}$ and organic acid were refluxed in absolute ethanol medium in a conical flask for about 30 minutes on a water bath. The clear solution cooled to get the characteristic colour precipitate of $\mathrm{Rb}$ or $\mathrm{Cs}$ metal salt. It was filtered, washed with solvent and dried in a electric oven at $80^{\circ} \mathrm{C}$. Stoichiometric amount of rubidium or caesium metal salt of organic acid \& thiosalicylic acid was refluxed in absolute ethanol for 1-2 hour with constant stirring, then cooled which led the coloured complex precipitated out. The precipitated complex was filtered washed with solvent and dried in electric oven at $80^{\circ} \mathrm{C}$.

\section{Results and Discussion}

Conductivity of the ligand, o-aminothiophenol and its mixed ligand complexes of rubidium \& Caesium metal salts of different organic acid were measured by Systronics Digital Reading Conductivity Meter-304 at $30^{\circ} \mathrm{C}$ in $10^{-3} \mathrm{M}$ DMF solution. The conductivity values are given Table-1 
Table 1

\begin{tabular}{|c|c|c|c|c|c|c|c|}
\hline \multirow{2}{*}{ Compound } & \multirow{2}{*}{ Colour } & \multirow{2}{*}{ M.p. Decomp. Trans. Temp. $\left({ }^{0} \mathrm{C}\right)$} & \multirow{2}{*}{ Conductivity } & \multicolumn{4}{|c|}{ Analysis \% (Found/Calcd.) } \\
\hline & & & & C & $\mathbf{H}$ & $\mathbf{N}$ & $\mathbf{M}$ \\
\hline O-aminothiophenol (OATP) & Colourless & $24 \mathrm{~m}$ & 5.0 & - & - & - & - \\
\hline $\mathrm{Rb}(1 \mathrm{~N} 2 \mathrm{~N})(\mathrm{OATP})$ & Deep brown & $205 \mathrm{~d}$ & 16.0 & $\begin{array}{c}50.0 \\
(50.26)\end{array}$ & $\begin{array}{c}3.44 \\
(3.35)\end{array}$ & $\begin{array}{c}7.56 \\
(7.52)\end{array}$ & $\begin{array}{c}21.30 \\
(22.25)\end{array}$ \\
\hline Cs $(1 \mathrm{~N} 2 \mathrm{~N})(\mathrm{OATP})$ & Deep brown & $193 \mathrm{t}$ & 20.0 & $\begin{array}{c}44.65 \\
(44.63) \\
\end{array}$ & $\begin{array}{c}3.05 \\
(3.07) \\
\end{array}$ & $\begin{array}{c}6.61 \\
(6.51) \\
\end{array}$ & $\begin{array}{c}22.30 \\
(22.23) \\
\end{array}$ \\
\hline Rb (8HQ) (OATP) & Light brown & $205 \mathrm{t}$ & 1.0 & $\begin{array}{c}50.91 \\
(50.83)\end{array}$ & $\begin{array}{c}3.70 \\
(3.67)\end{array}$ & $\begin{array}{c}7.95 \\
(7.70)\end{array}$ & $\begin{array}{c}24.06 \\
(24.01)\end{array}$ \\
\hline Cs (8HQ) (OATP) & Light brown & $200 \mathrm{~d}$ & 20.0 & $\begin{array}{c}44.81 \\
(44.75)\end{array}$ & $\begin{array}{c}2.30 \\
(3.23)\end{array}$ & $\begin{array}{c}6.99 \\
(6.96) \\
\end{array}$ & $\begin{array}{c}32.13 \\
(30.06)\end{array}$ \\
\hline $\mathrm{Rb}(0 \mathrm{NP})(\mathrm{OATP})$ & Brownish Yellow & $196 \mathrm{t}$ & 14.0 & $\begin{array}{c}41.42 \\
(41.36)\end{array}$ & $\begin{array}{c}3.20 \\
(3.16) \\
\end{array}$ & $\begin{array}{c}6.09 \\
(6.04)\end{array}$ & $\begin{array}{c}24.51 \\
(24.42)\end{array}$ \\
\hline Cs (0NP) (OATP) & Brownish Yellow & $190 \mathrm{t}$ & 15.0 & $\begin{array}{c}36.40 \\
(35.33)\end{array}$ & $\begin{array}{c}2.80 \\
(2.77)\end{array}$ & \begin{tabular}{|c|}
7.17 \\
$(7.04)$ \\
\end{tabular} & $\begin{array}{c}33.61 \\
(33.58) \\
\end{array}$ \\
\hline $\mathrm{Rb}$ (DNP) (OATP) & Brownish Yellow & $208 \mathrm{~d}$ & 10.0 & $\begin{array}{c}36.67 \\
(36.64) \\
\end{array}$ & \begin{tabular}{|c|}
2.58 \\
$(2.54)$ \\
\end{tabular} & $\begin{array}{c}10.71 \\
(10.68) \\
\end{array}$ & $\begin{array}{c}21.66 \\
(21.61) \\
\end{array}$ \\
\hline Gs (DNP) (OATP) & Brownish Yellow & $200 \mathrm{~d}$ & 14.0 & $\begin{array}{c}32.70 \\
(32.63) \\
\end{array}$ & $\begin{array}{c}2.30 \\
(2.24) \\
\end{array}$ & $\begin{array}{c}9.55 \\
(9.50) \\
\end{array}$ & $\begin{array}{c}30.21 \\
(30.14)\end{array}$ \\
\hline
\end{tabular}

*Molar conductivity $\mathrm{ohm}^{-1} \mathrm{~mol}^{-1} \mathrm{~cm}^{2}$ of $10^{-3} \mathrm{M}$ solution in $\mathrm{MeOH}$

Almost all complexes have been found to be coloured \& stable in dry air but decomposed in moist air. They are soluble in polar solvents e.g. $\mathrm{MeOH}, \mathrm{EtOH}, \mathrm{DMF}$ but insoluble in non-polar solvents. From result, it was evident that all these complexes either melting point of the ligand, indicating their greater thermal stability. Infrared spectral of the ligand (oaminothiophenol) and its hitherto un-known mixed ligand rubidium and caesium metal complexes of general formula, ML.HL', where $\mathrm{M}=\mathrm{Rb}$ or $\mathrm{Cs}, \mathrm{L}=$ deprotonated o-nitrophenol, 2, 4-dinitrophenol, 1-nitroso-2-naphthol, 8hydroxyquionoline \& $\mathrm{HL}^{\prime}=\mathrm{o}-$ aminothiophenol have been recorded in region $4000-650 \mathrm{~cm}^{-1}$ in $\mathrm{KBr}$ phase with the help of spectrophotometer. Pertinent IR data for these compounds were recorded in Table-2

Table 2

\begin{tabular}{|c|c|c|c|}
\hline \multirow{2}{*}{ Compound } & \multicolumn{3}{|c|}{ Selected IR absorption bands (in $\mathbf{~ c m}^{-\mathbf{1}}$ ) } \\
\cline { 2 - 4 } & $\mathbf{v}_{-} \mathbf{N}-\mathbf{H}$ & $\mathbf{v}_{\mathbf{S}}-\mathbf{H}$ & $\mathbf{v}_{-\mathbf{N H}} \mathbf{2}$ \\
\hline $\begin{array}{c}\text { O-aminothiophenol } \\
\text { (OATP) }\end{array}$ & $3200 \mathrm{~s}, 3100 \mathrm{~m}$ & $2350 \mathrm{~m}$ & $1490 \mathrm{~s}$ \\
\hline $\mathrm{Rb}(0 \mathrm{NP})$ OATP & $3170 \mathrm{~m}, 3075 \mathrm{~m}$ & $2300 \mathrm{~m}$ & $1400 \mathrm{~s}, 1360 \mathrm{~m}$ \\
\hline $\mathrm{Cs}(0 \mathrm{NP})$ OATP & $3170 \mathrm{~m}, 3075 \mathrm{~m}$ & $2320 \mathrm{~m}$ & $1400 \mathrm{~s}, 1370 \mathrm{~m}$ \\
\hline $\mathrm{Rb}(\mathrm{DNP})$ OATP & $3180 \mathrm{~s}, 3090 \mathrm{~m}$ & $2310 \mathrm{~m}$ & $1400 \mathrm{~s}, 1360 \mathrm{~m}$ \\
\hline $\mathrm{Cs}(\mathrm{dNP})$ OATP & $3170 \mathrm{~m}, 3070 \mathrm{~m}$ & $2300 \mathrm{~m}$ & $1400 \mathrm{~s}, 1360 \mathrm{~m}$ \\
\hline $\mathrm{Rb}(1 \mathrm{~N} 2 \mathrm{~N})$ OATP & $3150 \mathrm{~m}, 3070 \mathrm{~m}$ & $2300 \mathrm{~m}$ & $1400 \mathrm{~s}, 1375 \mathrm{~m}$ \\
\hline $\mathrm{Cs}(1 \mathrm{~N} 2 \mathrm{~N})$ OATP & $3170 \mathrm{~m}, 3075 \mathrm{~m}$ & $2290 \mathrm{~m}$ & $1400 \mathrm{~s}, 1380 \mathrm{~m}$ \\
\hline $\mathrm{Rb}(8 \mathrm{HQ})$ OATP & $3180 \mathrm{~m}, 3027 \mathrm{~m}$ & $2310 \mathrm{~m}$ & $1400 \mathrm{~s}, 1370 \mathrm{~m}$ \\
\hline $\mathrm{Cs}(8 \mathrm{HQ})$ OATP & $3150 \mathrm{~m}, 3080 \mathrm{~m}$ & $2100 \mathrm{~m}$ & $1400 \mathrm{~s}, 1380 \mathrm{~m}$ \\
\hline
\end{tabular}

$\mathrm{s}=$ strong, $\mathrm{w}=$ weak, $\mathrm{m}=$ medium, $\mathrm{sh}=$ shoulder

The absorption range of $\mathrm{N}-\mathrm{H}$ has been observed in the mixed ligand complexes of rubidium and caesium metals, which might be due to coordination of $-\mathrm{NH}_{2}$ group to the rubidium $\&$ caesium metals. As shown in fig.

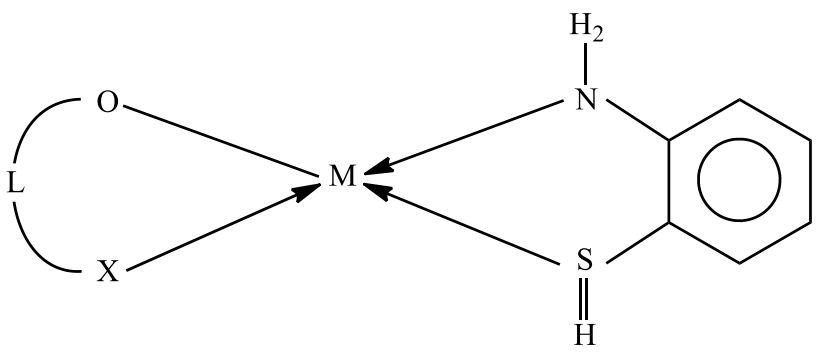

\section{Conclusion}

The spectrum of the ligand contains a moderately medium absorption band at $2550 \mathrm{~cm}^{-1}$ the region of $\mathrm{S}-\mathrm{H}$ vibration frequency, this band has shifted down by $\sim 40-70 \mathrm{~cm}^{-1}$ in the mixed ligand rubidium \& caesium metal complexes, indicating there by the coordination has taken place through $\mathrm{S}-$ atom of S-H group.

\section{References}

1. Livingston SE. J. Chem. Soc. 2016; 437:1042.

2. Olszewaski EJ, Albinak MJ. J. Inorg. Nucl. Chem. 2015; 27:1431.

3. Hieber W, Bruck R. Z. Anorg. Allgem. Chem. 2013; 13:269.

4. Stiefel RI. Waters, J.H., Billing, E. \& Gray, H.B.; J. Am. Chem. Soc. 2017; 87:3016.

5. Prakash D, Hussain SA, Yadav SP, Gupta AK. Asian J. Chem. 2019; 12(4):1135.

(where $\mathrm{M}=\mathrm{Rb}$ or $\mathrm{Cs}, \mathrm{X}=\mathrm{O}$ or $\mathrm{N}$ ) 